\title{
On the equivariant main conjecture of Iwasawa theory
}

\author{
by \\ Malte Witte (Leipzig)
}

Introduction. The main conjecture of Iwasawa theory for an abelian number field in its classical formulation describes the Galois module structure of the class groups in the limit over the intermediate fields of its cyclotomic $\mathbb{Z}_{p}$-extension. The eigenspace of this limit with respect to a Dirichlet character $\chi$ associated to the ground field is related to the corresponding $p$-adic $L$-function or to the eigenspace of the group of global units modulo cyclotomic units, depending on the parity of $\chi$. The conjecture was proved in 1984 by B. Mazur and A. Wiles [MW84].

Recently, D. Burns and C. Greither [BG03] deduced an equivariant version of the main conjecture as the key to their proof of the equivariant Tamagawa number conjecture. Here, "equivariant" refers to the fact that one retains the full Galois module structure instead of decomposing the modules by characters.

A. Huber and G. Kings [HK03] also use a variant of the Iwasawa main conjecture in their proof of the Tamagawa number conjecture for Dirichlet motives. It consists, like the classical formulation, of a separate statement for each Dirichlet character. In particular, it is weaker than the formulation in [BG03].

In the present article, we use this statement and the theorem of FerreroWashington to reprove the equivariant conjecture of [BG03] in a slightly more general form.

Fix an odd prime $p$ and let $\left(K_{\infty}, \varrho, U\right)$ be a triple consisting of the cyclotomic extension $K_{\infty}$ of an abelian number field, a one-dimensional representation $\varrho$ of $\mathrm{G}(\overline{\mathbb{Q}} / \mathbb{Q})$ on a finite extension $\mathcal{O}_{p}$ of $\mathbb{Z}_{p}$, and an open subscheme $U$ of Spec $\mathbb{Z}$, subject to the condition that the ramification index in $K_{\infty} / \mathbb{Q}$ of every point in $U$ is prime to $p$.

Like [BG03] and [HK03] we use continuous étale cohomology to assign to each of such triples a complex $R^{\bullet}=\mathbf{R} \Gamma_{\mathrm{Iw}}\left(U_{K_{\infty}}, \mathcal{O}_{p}(\varrho)\right)$ of modules over the

2000 Mathematics Subject Classification: Primary 11R23; Secondary 11R18, 19F27, $11 \mathrm{R} 42$. 
profinite group ring $\Omega=\mathcal{O}_{p} \llbracket \mathrm{G}\left(K_{\infty} / \mathbb{Q}\right) \rrbracket$. Further, we define a cyclotomic element $c\left(U_{K_{\infty}}, \varrho\right)$ in the first cohomology module of $R^{\bullet}$ and a $p$-adic $L$ element $L\left(U_{K_{\infty}}, \varrho^{-1} \varepsilon_{\text {cycl }}\right)$ in the quotient ring of $\Omega$. Here, $\varepsilon_{\text {cycl }}$ denotes the cyclotomic character. The quotient $R \bullet / \Omega c\left(U_{K_{\infty}}, \varrho\right)$ turns out to be a perfect complex that is torsion, i.e. acyclic after base change to the quotient ring. Note that this is no longer true if we drop the condition on the type of ramification in $U$.

Using the determinant functor of F. Knudsen and D. Mumford we can attach to each perfect torsion complex $P^{\bullet}$ an invertible fractional ideal of $\Omega$ called the characteristic ideal of $P^{\bullet}$. Our main result then reads as follows.

Theorem 0.1 (see Theorem 7.4).

(i) (Vanishing of the $\mu$-invariant) Let $\mathfrak{p}$ be a prime ideal of codimension 1 of $\Omega$, with $p \in \mathfrak{p}$. Then $\left(R^{\bullet} / \Omega c\left(U_{K_{\infty}}, \varrho\right)\right)_{\mathfrak{p}}$ is acyclic.

(ii) (Iwasawa main conjecture) $L\left(U_{K_{\infty}}, \varrho^{-1} \varepsilon_{\text {cycl }}\right)$ generates the characteristic ideal of $R^{\bullet} / \Omega c\left(U_{K_{\infty}}, \varrho\right)$.

The formulation of the main conjecture in [BG03] corresponds to Theorem 0.1(ii) for all triples $\left(\mathbb{Q}\left(\zeta_{n p} \infty\right), \varepsilon_{\text {cycl }}^{r}\right.$, Spec $\left.\mathbb{Z}[1 / n p]\right)$, the version in [HK03] to triples $\left(\mathbb{Q}_{\infty}, \chi \varepsilon_{\text {cycl }}^{r}\right.$, Spec $\left.\mathbb{Z}[1 / p]\right)$. Here, $r$ and $n$ are integers, $\mathbb{Q}_{\infty}$ is the $\mathbb{Z}_{p}$-extension of $\mathbb{Q}$ and $\chi$ is any Dirichlet character.

The relation to the classical Iwasawa main conjecture is established by the fact that the first and second cohomology modules of the complex $R^{\bullet} / \Omega c\left(U_{K_{\infty}}, \varrho\right)$ for $\varrho=\varepsilon_{\text {cycl }}$ are essentially given by the limit of the $p$-primary parts of the global units modulo cyclotomic units, respectively of the class groups, taken over the intermediate fields of $K_{\infty} / \mathbb{Q}$. Using $\widetilde{R} \bullet / c\left(U_{K_{\infty}}, \varrho\right)$ in lieu of these classical objects leads to a smoother formulation of the conjecture that circumvents the problems usually connected to $p$ dividing the order of $\mathrm{G}(K / \mathbb{Q})$ (see the discussion in $[\mathrm{HK} 03]$ ).

The main idea of the proof of Theorem 0.1 is essentially the same as in [BG03]. However, we can clarify the argument considerably by using the result of [HK03]. Originally, D. Burns and C. Greither derived their theorem from the result of B. Mazur and A. Wiles. This approach necessitates some rather involved deduction steps to deal with the first cohomology group of $R^{\bullet} / \Omega c\left(U_{K_{\infty}}, \varrho\right)$, in particular for Theorem 0.1(i). (Note that this step of the argument in [BG03] contains an inaccuracy that was later corrected in the appendix of [Fla04].) The additional strength of the main conjecture in [HK03] allows us to present a comparatively quick proof of this part of the theorem. Recall that A. Huber and G. Kings do not use the result of [MW84]. Instead, they give an independent proof of their statement, using the Euler system approach of V. A. Kolyvagin and K. Rubin [Rub00].

As [BG03] and [HK03], we do not treat the case $p=2$, but this gap has meanwhile been filled by M. Flach in [Fla04]. 
The article is organised as follows. In Section 1 we introduce the characteristic ideal of a perfect torsion complex. Section 2 consists of a collection of algebraic properties of $\Omega$ that turn out to be useful in the later sections.

The definition of the complex $\mathbf{R} \Gamma_{\mathrm{Iw}}\left(U_{K_{\infty}}, \mathcal{O}_{p}(\varrho)\right)$ is given in Section 3. In the subsequent section we calculate its cohomology modules in the special case $\varrho=\varepsilon_{\text {cycl }}$, which is closely related to classical Iwasawa theory.

To deal with the ramification of $\varrho$ we need an explicit description of the relative cohomology modules associated to closed subschemes of $U$. This is achieved in Section 5 .

In Section 6 we extend the classical construction of cyclotomic elements and $L$-elements to our setting. The final section is devoted to the proof of the main theorem.

Acknowledgements. The paper was partially written up during a visit at the Max Planck Institute for Mathematics in Bonn. The author would like to thank this institution for its hospitality and support. Before all others, he wishes to express his gratitude to A. Huber for introducing him to this subject and for numerous valuable discussions.

1. Characteristic ideals. The notion of the characteristic ideal of a perfect torsion complex is a variant of the usual determinant functor of F. Knudsen and D. Mumford [KM76]. It is less flexible than the latter, but easier to handle.

Let $R$ be any commutative ring (with unit) and denote by $Q(R)$ the total ring of fractions of $R$. Further, we write $\mathfrak{I}(R)$ for the abelian group of invertible fractional ideals, i.e. $R$-submodules $I$ of $Q(R)$ which are locally free of rank 1 and which satisfy $Q(R) \otimes_{R} I=Q(R)$.

We can view $\mathfrak{I}$ as a functor from the category of commutative rings to abelian groups if we restrict the morphisms of the former to the following class.

Definition 1.1. We call a ring homomorphism $\phi: R \rightarrow S$ extendable if it extends to a homomorphism $Q(R) \rightarrow Q(S)$, also denoted by $\phi$.

Examples of extendable homomorphisms include all flat homomorphisms and all integral extensions.

If $\phi: R \rightarrow S$ is extendable, then $\mathfrak{I}(\phi)$ is given by

$$
\mathfrak{I}(\phi)(I)=\phi(I) S
$$

for all $I \in \mathfrak{I}(R)$.

Assume that $R$ is noetherian. Then an element of $\mathfrak{I}(R)$ is uniquely determined by the following local conditions.

Proposition 1.2. Let $R$ be noetherian and $I, J \in \mathfrak{I}(R)$. Then $I=J$ if and only if $I R_{\mathfrak{p}}=J R_{\mathfrak{p}}$ for all non-zero divisors $r$ and all primes $\mathfrak{p}$ associated 
to $R / r R$. These are exactly the primes of codimension 1 if $R$ is CohenMacaulay.

Proof. This follows by the same argument as [Eis99, Prop. 11.3].

Definition 1.3. We call a complex $P^{\bullet}$ of $R$-modules a torsion complex if $Q(R) \otimes_{R} P^{\bullet}$ is acyclic. $P^{\bullet}$ is called perfect if it is quasi-isomorphic to a bounded complex of finitely generated projective $R$-modules.

Let $\operatorname{det}_{R} P^{\bullet}$ denote the determinant of $P^{\bullet}$ according to F. Knudsen and D. Mumford [KM76]. If $P^{\bullet}$ is a perfect torsion complex, then the natural isomorphism

$$
Q(R) \otimes_{R} \operatorname{det}_{R} P^{\bullet}=Q(R)
$$

allows us to view $\operatorname{det}_{R} P^{\bullet}$ as an invertible fractional ideal of $R$.

Definition 1.4. We call char $P^{\bullet}=\left(\operatorname{det}_{R} P^{\bullet}\right)^{-1} \in \mathfrak{I}(R)$ the characteristic ideal of $P^{\bullet}$.

The characteristic ideal enjoys the following properties.

Proposition 1.5. Let $P^{\bullet}$ be a perfect torsion complex of $R$-modules.

(i) char $P^{\bullet}$ depends only on the quasi-isomorphism class of $P^{\bullet}$.

(ii) $\operatorname{char} P^{\bullet}[1]=\left(\operatorname{char} P^{\bullet}\right)^{-1}$.

(iii) If $P_{1}^{\bullet} \rightarrow P_{2}^{\bullet} \rightarrow P_{3}^{\bullet}$ is a distinguished triangle of perfect torsion complexes in the derived category, then

$$
\operatorname{char} P_{2}^{\bullet}=\operatorname{char} P_{1}^{\bullet} \operatorname{char} P_{3}^{\bullet} .
$$

(iv) If $\phi: R \rightarrow S$ is an extendable homomorphism, then $\mathbf{L} \phi_{*}\left(P^{\bullet}\right)=$ $S \otimes \frac{\mathbb{L}}{R} P^{\bullet}$ is a perfect torsion complex of $S$-modules and

$$
\operatorname{char} \mathbf{L} \phi_{*}\left(P^{\bullet}\right)=\mathfrak{I}(\phi)\left(\operatorname{char} P^{\bullet}\right) .
$$

(v) If the cohomology modules of $P^{\bullet}$ are themselves perfect, i.e. of finite Tor-dimension, then

$$
\operatorname{char} P^{\bullet}=\prod_{n \in \mathbb{Z}}\left(\operatorname{char} \mathrm{H}^{n} P^{\bullet}\right)^{(-1)^{n}} .
$$

(vi) If $R$ is a noetherian and normal domain and $M$ any torsion module of finite projective dimension (considered as complex concentrated in degree 0$)$, then char $M$ coincides with the content of $M$, as defined in [Bou89, VII, §4.5]. In particular, if $R=\mathbb{Z}_{p} \llbracket T \rrbracket$, then char $M$ is the characteristic ideal of Iwasawa theory.

Proof. Everything follows easily from the corresponding properties of the determinant functor, as given in [KM76].

REMARK 1.6. If $R$ is not reduced, then the usual determinant functor is additive only for the class of "true" triangles. In the following, we will only consider reduced rings. However, note that in our setting, (iii) is indeed true 
for arbitrary distinguished triangles. The reason is that one can always replace the distinguished triangle by a true triangle of strictly perfect torsion complexes, the particular choice of which, according to (i), does not matter. For the determinant functor, it is this non-canonical choice that causes trouble.

REMARK 1.7. One can also deduce Proposition 1.5 from the results of [BB05] on the more sophisticated notion of the refined Euler characteristic. To this end, note that for a perfect torsion complex $P^{\bullet}$, the only trivialisation is the zero map and char $P^{\bullet}$ is the image of $-\chi\left(P^{\bullet}, 0\right)$ under the natural homomorphism $K_{0}(R, Q(R)) \rightarrow \mathfrak{I}(R)$.

2. The profinite group ring of a $\mathbb{Z}_{p}$-extension. In this section we will assemble some useful facts about cyclotomic $\mathbb{Z}_{p}$-extensions and profinite group rings. A large part of the material can also be found in [BG03, §6.1].

Throughout this article, $p$ will denote a fixed odd prime. Let $\mathbb{Q}_{\infty}$ be the unique $\mathbb{Z}_{p}$-extension of $\mathbb{Q}$. The cyclotomic $\mathbb{Z}_{p}$-extension of a number field is given by $K_{\infty}=K \mathbb{Q}_{\infty}$. We shall always make the additional assumption that $K$ is an abelian extension of $\mathbb{Q}$. The theorem of Kronecker-Weber then shows that there exists the following distinguished choice of subfields of $K_{\infty}$.

Definition 2.1. Let $K_{0} \subset K_{\infty}$ be the subfield that is uniquely determined by the following two properties:

(i) $\mathrm{G}\left(K_{\infty} / \mathbb{Q}\right)=\mathrm{G}\left(K_{0} / \mathbb{Q}\right) \times \mathrm{G}\left(\mathbb{Q}_{\infty} / \mathbb{Q}\right)$,

(ii) $p^{2}$ does not divide the conductor of $K_{0}$.

We write $K_{n}$ for the subfield of $K_{\infty}$ of degree $p^{n}$ over $K_{0}$.

Let $\mathcal{O}_{p}$ be the valuation ring of an arbitrary finite extension of $\mathbb{Q}_{p}$ and write

$$
\Omega=\mathcal{O}_{p} \llbracket \mathrm{G}\left(K_{\infty} / \mathbb{Q}\right) \rrbracket=\lim _{n} \mathcal{O}_{p}\left[\mathrm{G}\left(K_{n} / \mathbb{Q}\right)\right]
$$

for the profinite group ring with coefficients in $\mathcal{O}_{p}$. Assume for simplicity that $\mathcal{O}_{p}$ contains all values of the characters of $\mathrm{G}\left(K_{0} / \mathbb{Q}\right)$. If $P_{\infty}$ is the maximal $p$-extension of $\mathbb{Q}$ inside $K_{\infty}$, then

$$
\Omega \cong \prod_{\theta} \mathcal{O}_{p} \llbracket \mathrm{G}\left(P_{\infty} / \mathbb{Q}\right) \rrbracket,
$$

where the product runs through the characters $\theta$ of $\mathrm{G}\left(K_{\infty} / P_{\infty}\right)$. Observe that $\mathcal{O}_{p} \llbracket \mathrm{G}\left(P_{\infty} / \mathbb{Q}\right) \rrbracket$ is a local Cohen-Macaulay ring of Krull dimension 2, but it is not regular unless $P_{\infty}=\mathbb{Q}_{\infty}$.

The normalisation of $\Omega$ in its total quotient ring $Q(\Omega)$ is given by

$$
\widetilde{\Omega} \cong \prod_{\chi} \mathcal{O}_{p} \llbracket \mathrm{G}\left(\mathbb{Q}_{\infty} / \mathbb{Q}\right) \rrbracket .
$$


Here, $\chi$ runs through the characters of $\mathrm{G}\left(K_{0} / \mathbb{Q}\right)$. Note that

$$
\Omega[1 / p]=\widetilde{\Omega}[1 / p] .
$$

The prime ideals $\mathfrak{p}$ of codimension 1 of $\Omega$ with $p \in \mathfrak{p}$ play a special role in our considerations. Recall that a torsion module $M$ over $\mathbb{Z}_{p} \llbracket T \rrbracket$ has vanishing Iwasawa $\mu$-invariant if $M$ is finitely generated as $\mathbb{Z}_{p}$-module. We generalise this as follows.

Lemma 2.2. Let $M$ be an $\Omega$-module which is finitely generated as $\mathcal{O}_{p^{-}}$ module. Then $M_{\mathfrak{p}}=0$ for all prime ideals $\mathfrak{p}$ of codimension 1 containing $p$.

Proof. We can view $M$ as a module over

$$
\mathbb{Z}_{p} \llbracket T \rrbracket \cong \mathbb{Z}_{p} \llbracket \mathrm{G}\left(K_{\infty} / K_{0}\right) \rrbracket
$$

via the natural inclusion

$$
i: \mathbb{Z}_{p} \llbracket \mathrm{G}\left(K_{\infty} / K_{0}\right) \rrbracket \hookrightarrow \mathcal{O}_{p} \llbracket \mathrm{G}\left(K_{\infty} / \mathbb{Q}\right) \rrbracket .
$$

The structure theorem for $\mathbb{Z}_{p} \llbracket T \rrbracket$-modules ([Was97, Prop. 13.19]) shows that $M_{(p)}=0$, since $\mathbb{Z}_{p} \llbracket T \rrbracket /\left(p^{n}\right)$ is not finitely generated over $\mathbb{Z}_{p}$ for integers $n \geq 0$. The statement for $\mathcal{O}_{p} \llbracket \mathrm{G}\left(K_{\infty} / \mathbb{Q}\right) \rrbracket$ follows because $i^{-1}(\mathfrak{p})=(p)$.

We will now determine the group of invertible ideals of $\Omega$. Since $\Omega$ is semilocal, it is given by

$$
\Im(\Omega)=Q(\Omega)^{\times} / \Omega^{\times} .
$$

In our main statement we compare two elements of $\mathfrak{I}(\Omega)$. If $\mathcal{O}_{p}^{\prime}$ is a faithfully flat extension of $\mathcal{O}_{p}$, e.g. the valuation ring of a finite extension of $Q\left(\mathcal{O}_{p}\right)$, then the induced map

$$
\mathfrak{I}(\Omega) \rightarrow \mathfrak{I}\left(\mathcal{O}_{p}^{\prime} \otimes_{\mathcal{O}_{p}} \Omega\right)
$$

is injective. Therefore, the above assumption that $\mathcal{O}_{p}$ contains the values of the characters of $\mathrm{G}\left(K_{\infty} / \mathbb{Q}\right)$ is no restriction for our purposes. (Alternatively, it can be circumvented by using components instead of characters, as in [MW84].)

If $\mathbb{Q}_{\infty} \subset L_{\infty} \subset K_{\infty}$ is any intermediate extension, we write

$$
\psi_{K_{\infty} / L_{\infty}}: \mathcal{O}_{p} \llbracket \mathrm{G}\left(K_{\infty} / \mathbb{Q}\right) \rrbracket \rightarrow \mathcal{O}_{p} \llbracket \mathrm{G}\left(L_{\infty} / \mathbb{Q}\right) \rrbracket
$$

for the natural projection. Note that the ring homomorphism $\psi_{K_{\infty} / L_{\infty}}$ is extendable. Indeed, the induced map $\widetilde{\psi}$ between the normalisations of both rings is extendable for almost trivial reasons. Since the inclusion $\Omega \rightarrow \widetilde{\Omega}$ is extendable and maps zero divisors to zero divisors, it follows that $\psi$ is extendable as well.

3. Iwasawa cohomology. Consider an open subscheme $U$ of $\operatorname{Spec} \mathbb{Z}$ and let $S$ denote its closed complement. If $F / \mathbb{Q}$ is a finite field extension, 
we set

$$
U_{F}=U \times \operatorname{Spec} \mathcal{O}_{F}, \quad S_{F}=S \times \operatorname{Spec} \mathcal{O}_{F},
$$

where $\mathcal{O}_{F}$ denotes the ring of integers of $F$. Write

$$
j_{F}: \operatorname{Spec} F \rightarrow U_{F}
$$

for the inclusion of the generic point. As before, $K_{\infty}$ will denote the cyclotomic $\mathbb{Z}_{p}$-extension of an abelian number field.

Let $M(\varrho)$ be a finitely generated $\mathcal{O}_{p}$-module $M$ together with a continuous representation

$$
\varrho: \mathrm{G}(\overline{\mathbb{Q}} / \mathbb{Q}) \rightarrow \operatorname{Aut}_{\mathcal{O}_{p}} M
$$

(where we give $\operatorname{Aut}_{\mathcal{O}_{p}} M$ its profinite topology). Let further

$$
\iota: \mathrm{G}(\overline{\mathbb{Q}} / \mathbb{Q}) \rightarrow \Omega^{\times}, \quad \iota(g)=\bar{g}^{-1} \in \mathrm{G}\left(K_{\infty} / \mathbb{Q}\right)
$$

denote the contragredient of the natural representation. The $\Omega[\mathrm{G}(\overline{\mathbb{Q}} / \mathbb{Q})]$ module

$$
\operatorname{Ind}_{K_{\infty} / \mathbb{Q}} M(\varrho)=\Omega(\iota) \otimes \mathcal{O}_{p} M(\varrho)
$$

gives rise to a projective system of étale sheaves

$$
j_{\mathbb{Q} *} \operatorname{Ind}_{K_{\infty} / \mathbb{Q}} M(\varrho)=\left(j_{\mathbb{Q} *}\left(\mathcal{O}_{p} / p^{n} \mathcal{O}_{p}\left[\mathrm{G}\left(K_{n} / \mathbb{Q}\right)\right](\iota) \otimes \mathcal{O}_{p} M(\varrho)\right)\right)_{n=1}^{\infty}
$$

on $U$. (We reemphasise that the action of $\mathrm{G}(\overline{\mathbb{Q}} / \mathbb{Q})$ on the module $\mathcal{O}_{p} / p^{n} \mathcal{O}_{p}\left[\mathrm{G}\left(K_{n} / \mathbb{Q}\right)\right](\iota)$ is given by $\iota$, i.e. $\mathcal{O}_{p} / p^{n} \mathcal{O}_{p}\left[\mathrm{G}\left(K_{n} / \mathbb{Q}\right)\right]$ is considered as trivial $\mathrm{G}(\overline{\mathbb{Q}} / \mathbb{Q})$-module. $)$

Definition 3.1. We define the Iwasawa complex of $M(\varrho)$ over $U$ to be the cochain complex of continuous étale cohomology

$$
\mathbf{R} \Gamma_{\text {Iw }}\left(U_{K_{\infty}}, M(\varrho)\right)=\mathbf{R}\left({\underset{\leftarrow}{n}}_{\lim _{\text {ét }}} \Gamma\left(U, j_{\mathbb{Q} *} \operatorname{Ind}_{K_{\infty} / \mathbb{Q}} M(\varrho)\right),\right.
$$

as constructed by U. Jannsen in [Jan88]. If $Z$ is a closed subscheme of $U$, we define

$$
\mathbf{R} \Gamma_{\mathrm{Iw}}\left(U_{K_{\infty}}, Z, M(\varrho)\right)=\mathbf{R}\left(\stackrel{\lim }{n}_{\Gamma_{\text {ét }}}\right)\left(U, Z, j_{\mathbb{Q} *} \operatorname{Ind}_{K_{\infty} / \mathbb{Q}} M(\varrho)\right)
$$

to be the complex of continuous étale cohomology with support in $Z$. These complexes are to be understood as objects of the derived category of $\Omega$ modules. Their $i$ th cohomology modules are denoted by $\mathrm{H}_{\mathrm{Iw}}^{i}\left(U_{K_{\infty}}, M(\varrho)\right)$, respectively $\mathrm{H}_{\mathrm{Iw}}^{i}\left(U_{K_{\infty}}, Z, M(\varrho)\right)$.

REMARK 3.2. Alternatively, it should also be possible to use the formalism of T. Ekedahl [Eke90].

Here are some basic properties of $\mathbf{R} \Gamma_{\mathrm{Iw}}\left(U_{K_{\infty}}, M(\varrho)\right)$. 
Proposition 3.3. Assume $p \notin U$.

(i) For all $i \in \mathbb{Z}$,

$$
\mathrm{H}_{\mathrm{IW}}^{i}\left(U_{K_{\infty}}, M(\varrho)\right)={\underset{n}{\longleftarrow}}_{\lim _{\mathrm{et}}} \mathrm{H}_{\mathrm{e}}^{i}\left(U_{K_{n}}, j_{K_{n} *} M(\varrho)\right),
$$

where the limit is taken with respect to the corestriction maps.

(ii) In particular,

$$
\mathrm{H}_{\mathrm{Iw}}^{0}\left(U_{K_{\infty}}, M(\varrho)\right)=0 .
$$

Proof. By [Mil86, Theorem II.2.13] the modules $\mathrm{H}_{\text {ét }}^{i}\left(U_{K_{n}}, j_{K_{n} *} M(\varrho)\right)$ are finite. The asserted equality in (i) follows by [Jan88, Proposition 1.6 and Lemma 1.15].

It remains to verify that $\mathrm{H}_{\mathrm{Iw}}^{0}\left(U_{K_{\infty}}, M(\varrho)\right)=0$. As $M$ is noetherian, there is an $n_{0}$ such that the inflation map

$$
\begin{aligned}
& \mathrm{H}_{\text {ét }}^{0}\left(U_{K_{n_{0}}}, j_{K_{n_{0}} *} M(\varrho)\right)=M(\varrho)^{\mathrm{G}\left(\overline{\mathbb{Q}} / K_{n_{0}}\right)} \\
& \qquad M(\varrho)^{\mathrm{G}\left(\overline{\mathbb{Q}} / K_{n}\right)}=\mathrm{H}_{\text {ét }}^{0}\left(U_{K_{n}}, j_{K_{n} *} M(\varrho)\right)
\end{aligned}
$$

is the identity for $n \geq n_{0}$, and therefore, the corestriction map is multiplication by $p^{n-n_{0}}$. Hence, the limit over the corestriction maps vanishes.

If both $U_{K_{\infty}}$ and $\varrho$ are unramified over $U$, then all sheaves in the projective system $j_{\mathbb{Q} *} \operatorname{Ind}_{K_{\infty} / \mathbb{Q}} M(\varrho)$ are locally constant. Under these circumstances one can identify $\mathbf{R} \Gamma_{\mathrm{Iw}}\left(U_{K_{\infty}}, M(\varrho)\right)$ with the complex of continuous cochains of the topological $\pi_{1}^{\text {ét }}(U)$-module $\operatorname{Ind}_{K_{\infty} / \mathbb{Q}} M(\varrho)$, where $\pi_{1}^{\text {ét }}(U)$ denotes the étale fundamental group of $U$ (see Proposition II.2.9 of [Mil86]). This setting has been extensively explored by J. Nekovár in [Nek03]. We recall some of the consequences.

Proposition 3.4. Assume that $U_{K_{\infty}}$ and $\varrho$ are unramified over $U$. Then $\mathbf{R} \Gamma_{\mathrm{Iw}}\left(U_{K_{\infty}}, M(\varrho)\right)$ is acyclic outside degrees 1 and 2.

Proof. The cohomological $p$-dimension of $\pi_{1}^{\text {ét }}(U)$ is 2 (see [NSW00, Theorem 8.3.19]).

Proposition 3.5. Let $M(\varrho)$ be free as an $\mathcal{O}_{p}$-module and let $W$ be a finitely generated $\Omega$-module. Assume that $U_{K_{\infty}}$ and $\varrho$ are unramified over $U$. Then there exists a natural quasi-isomorphism

$$
W \otimes_{\Omega}^{\mathbb{L}} \mathbf{R} \Gamma_{\mathrm{Iw}}\left(U_{K_{\infty}}, M(\varrho)\right)=\mathbf{R}\left(\stackrel{\lim }{n}_{\text {ét }}\right)\left(U, j_{\mathbb{Q} *}\left(W \otimes_{\Omega} \operatorname{Ind}_{K_{\infty} / \mathbb{Q}} M(\varrho)\right)\right) .
$$

Proof. See [Nek03, Proposition 3.4.4].

Observe that for any $\varrho: \mathrm{G}(\overline{\mathbb{Q}} / \mathbb{Q}) \rightarrow \mathcal{O}_{p}^{\times}$we can find an abelian cyclotomic $\mathbb{Z}_{p}$-extension $K_{\infty}$ such that $\varrho$ factors through $\mathrm{G}\left(K_{\infty} / \mathbb{Q}\right)$. We will then denote by

$$
\mathrm{Tw}_{\varrho}: \mathcal{O}_{p} \llbracket \mathrm{G}\left(K_{\infty} / \mathbb{Q}\right) \rrbracket \rightarrow \mathcal{O}_{p} \llbracket \mathrm{G}\left(K_{\infty} / \mathbb{Q}\right) \rrbracket
$$


the ring automorphism that maps $g \in \mathrm{G}\left(K_{\infty} / \mathbb{Q}\right)$ to $\varrho(g) g$. For any ring homomorphism $f: R \rightarrow S$ and any $R$-module $M$ we write

$$
f_{*} M=S \otimes_{R} M
$$

for the base extension to $S$.

Proposition 3.6. Let $M(\varrho)$ be free as an $\mathcal{O}_{p}$-module. Assume that $\varrho$ is unramified outside a finite set of primes. Choose $U$ such that for any $l \in U$ the ramification index of $l$ in $K_{\infty} / \mathbb{Q}$ is prime to $p$. Then:

(i) $\mathbf{R} \Gamma_{\mathrm{Iw}}\left(U_{K_{\infty}}, M(\varrho)\right)$ is perfect.

(ii) For any intermediate field $\mathbb{Q}_{\infty} \subset L_{\infty} \subset K_{\infty}$ there exists a natural quasi-isomorphism

$$
\mathbf{L} \psi_{K_{\infty} / L_{\infty} *} \mathbf{R} \Gamma_{\mathrm{Iw}}\left(U_{K_{\infty}}, M(\varrho)\right)=\mathbf{R} \Gamma_{\mathrm{Iw}}\left(U_{L_{\infty}}, M(\varrho)\right) .
$$

(iii) For any $\chi: \mathrm{G}\left(K_{\infty} / \mathbb{Q}\right) \rightarrow \mathcal{O}_{p}^{\times}$there exist a natural quasi-isomorphism

$$
\mathrm{Tw}_{\chi *} \mathbf{R} \Gamma_{\mathrm{Iw}}\left(U_{K_{\infty}}, M(\varrho)\right)=\mathbf{R} \Gamma_{\mathrm{Iw}}\left(U_{K_{\infty}}, M\left(\chi^{-1} \varrho\right)\right) .
$$

Proof. Let $V$ be an open subscheme of $U$ such that both $\varrho$ and $V_{K_{\infty}}$ are unramified over $U$. The localisation triangle

$$
\mathbf{R} \Gamma_{\mathrm{Iw}}\left(U_{K_{\infty}}, U-V, M(\varrho)\right) \rightarrow \mathbf{R} \Gamma_{\mathrm{Iw}}\left(U_{K_{\infty}}, M(\varrho)\right) \rightarrow \mathbf{R} \Gamma_{\mathrm{Iw}}\left(V_{K_{\infty}}, M(\varrho)\right)
$$

([Jan88, 3.6]) implies that to prove (i), it is sufficient to show that the two outer complexes are perfect. The right complex is immediately seen to be perfect by Proposition 3.5. We will prove in Proposition 5.1 that the left complex is perfect as well.

By Remark 5.3 and the localisation triangle it also suffices to prove (ii) and (iii) for the scheme $V$. Claim (ii) then follows directly from the above proposition. For (iii) it remains to notice that

$$
\begin{aligned}
\operatorname{Tw}_{\chi^{*}} \operatorname{Ind}_{K_{\infty} / \mathbb{Q}} M(\varrho) & \rightarrow \operatorname{Ind}_{K_{\infty} / \mathbb{Q}} M\left(\chi^{-1} \varrho\right), \\
1 \otimes w \otimes m & \mapsto \operatorname{Tw}_{\chi}(w) \otimes m \quad(w \in \Omega(\iota), m \in M(\varrho))
\end{aligned}
$$

is a $\mathrm{G}(\overline{\mathbb{Q}} / \mathbb{Q})$-equivariant isomorphism of $\Omega$-modules.

4. Cohomology of $\mathcal{O}_{p}\left(\varepsilon_{\text {cycl }}\right)$. In this section we calculate the cohomology of the one-dimensional representation $\mathcal{O}_{p}\left(\varepsilon_{\text {cycl }}\right)$ given by the cyclotomic character

$$
\varepsilon_{\text {cycl }}: \mathrm{G}(\overline{\mathbb{Q}} / \mathbb{Q}) \rightarrow \mathbb{Z}_{p}^{\times}
$$

The following proposition establishes the link to the objects of classical Iwasawa theory.

Proposition 4.1. Let $U$ be an open subscheme of $X=\operatorname{Spec} \mathbb{Z}$ such that $p$ lies in the complement $S$ of $U$. 
(i) There exists a canonical isomorphism of $\Omega$-modules

$$
\mathrm{H}_{\mathrm{Iw}}^{1}\left(U_{K_{\infty}}, \mathcal{O}_{p}\left(\varepsilon_{\text {cycl }}\right)\right)={\underset{n}{\lim }}_{n} \mathcal{O}_{p} \otimes_{\mathbb{Z}} \mathbb{G}_{m}\left(U_{K_{n}}\right),
$$

where $\mathbb{G}_{m}$ denotes the multiplicative group.

(ii) The following sequence of $\Omega$-modules is exact:

$$
\begin{aligned}
0 & \rightarrow \underset{n}{\lim _{n}} \mathcal{O}_{p} \otimes_{\mathbb{Z}} \mathbb{G}_{m}\left(X_{K_{n}}\right) \rightarrow \mathrm{H}_{\mathrm{Iw}}^{1}\left(U_{K_{\infty}}, \mathcal{O}_{p}\left(\varepsilon_{\text {cycl }}\right)\right) \\
& \rightarrow \underset{n}{\lim } \mathrm{H}_{\text {ét }}^{0}\left(S_{K_{n}}, \mathcal{O}_{p}\right) \rightarrow \underset{n}{\lim } \mathcal{O}_{p} \otimes_{\mathbb{Z}} \operatorname{Pic}\left(X_{K_{n}}\right) \\
& \rightarrow \mathrm{H}_{\mathrm{Iw}}^{2}\left(U_{K_{\infty}}, \mathcal{O}_{p}\left(\varepsilon_{\text {cycl }}\right)\right) \rightarrow \underbrace{\lim }_{n} \mathrm{H}_{\text {ét }}^{1}\left(S_{K_{n}}, \mathcal{O}_{p}\right) \rightarrow \mathcal{O}_{p} \rightarrow 0 .
\end{aligned}
$$

Proof. This is proved in the same way as [BG03, Proposition 5.1]. The idea is to combine the calculation of the cohomology groups of $\mathbb{G}_{m}$ in [Mil86, Proposition II.2.1] with the Kummer exact sequence on $U_{K_{n}}$ and then to pass to the limit. The last term can then be identified as the tensor product of

$$
\mathbb{Z}_{p}=\lim _{n} \operatorname{Ker}\left(\mathrm{H}_{\text {ét }}^{3}\left(X_{K_{n}}, \mathbb{G}_{m}\right) \stackrel{p^{n}}{\longrightarrow} \mathrm{H}_{\text {ét }}^{3}\left(X_{K_{n}}, \mathbb{G}_{m}\right)\right)
$$

with $\mathcal{O}_{p}$.

This result is complemented by the following

Proposition 4.2. There exist (non-canonical) isomorphisms of $\Omega$-modules

$$
\begin{aligned}
& \lim _{\hbar} \mathrm{H}_{\text {ét }}^{0}\left(S_{K_{n}}, \mathcal{O}_{p}\right) \cong \mathcal{O}_{p}\left[\mathrm{G}\left(K_{\infty} / \mathbb{Q}\right) / D_{p}\right], \\
& \lim _{n} \mathrm{H}_{\text {ét }}^{1}\left(S_{K_{n}}, \mathcal{O}_{p}\right) \cong \bigoplus_{l \in S} \mathcal{O}_{p}\left[\mathrm{G}\left(K_{\infty} / \mathbb{Q}\right) / D_{l}\right],
\end{aligned}
$$

where $D_{l}$ is the decomposition subgroup of the prime $l$ in $\mathrm{G}\left(K_{\infty} / \mathbb{Q}\right)$.

Proof. One of the fundamental properties of $\mathbb{Z}_{p}$-extensions is the fact that $K_{\infty} / K_{0}$ is unramified outside the primes over $p$ (see [Was97, Prop. 13.2]). For cyclotomic $\mathbb{Z}_{p}$-extensions one also knows that there exists a number $n_{0}$ such that all primes over $p$ are totally ramified in $K_{\infty} / K_{n_{0}}$ and such that none of the primes in $S_{K_{n_{0}}}$ splits in $K_{\infty} / K_{n_{0}}$ (see [Was97, Ex. 13.2]). In particular, $S_{K_{\infty}} \rightarrow S_{K_{n}}$ is a homeomorphism for $n \geq n_{0}$.

On the other hand,

$$
\mathrm{H}_{\text {ét }}^{0}\left(S_{K_{n}}, \mathcal{O}_{p}\right) \cong \bigoplus_{v \in S_{K_{n}}} \mathcal{O}_{p} \cong \mathrm{H}_{\text {ét }}^{1}\left(S_{K_{n}}, \mathcal{O}_{p}\right) .
$$

An elementary calculation shows that the corestriction map

$$
\mathrm{H}_{\text {ét }}^{i}\left(S_{K_{n+1}}, \mathcal{O}_{p}\right) \rightarrow \mathrm{H}_{\text {ét }}^{i}\left(S_{K_{n}}, \mathcal{O}_{p}\right)
$$

for $n \geq n_{0}$ is the identity for $i=1$, and is the multiplication by the residue degree of $v$ on the $v$-component of $\mathrm{H}_{\text {ét }}^{0}\left(S_{K_{n+1}}, \mathcal{O}_{p}\right)$ for $i=0$. But the residue 
degree is 1 or $p$ depending on whether $v$ lies over $p$ or not. Now pass to the limit. The choice of an element of $S_{K_{\infty}}$ for each prime in $S$ induces the desired isomorphisms.

Corollary 4.3. Let $T$ be a closed subscheme of $U$. Then the complex $\mathbf{R} \Gamma_{\mathrm{Iw}}\left(U_{K_{\infty}}, T, \mathcal{O}_{p}\left(\varepsilon_{\text {cycl }}\right)\right)$ is acyclic outside degree 3 and

$$
\mathrm{H}_{\mathrm{Iw}}^{3}\left(U_{K_{\infty}}, T, \mathcal{O}_{p}\left(\varepsilon_{\text {cycl }}\right)\right) \cong \bigoplus_{l \in T} \mathcal{O}_{p}\left[\mathrm{G}\left(K_{\infty} / \mathbb{Q}\right) / D_{l}\right] .
$$

Proof. Easy application of the snake lemma.

5. Local factors. In this section we examine the relative cohomology complexes $\mathbf{R} \Gamma_{\mathrm{Iw}}\left(U_{K_{\infty}}, S, M(\varrho)\right)$ for arbitrary continuous $\mathrm{G}(\overline{\mathbb{Q}} / \mathbb{Q})$-representations $M(\varrho)$, where $M$ is any finitely generated $\mathcal{O}_{p}$-module. This will also complete the proof of Proposition 3.6. Our aim is to extend Corollary 4.3 as follows.

Proposition 5.1. Let $U$ be an open subscheme of $\operatorname{Spec} \mathbb{Z}[1 / p], S$ a closed subscheme of $U$. Then:

(i) $\mathbf{R} \Gamma_{\mathrm{Iw}}\left(U_{K_{\infty}}, S, M(\varrho)\right)$ is acyclic outside degree 3 .

(ii) $\mathrm{H}_{\mathrm{Iw}}^{3}\left(U_{K_{\infty}}, S, M(\varrho)\right)$ is a finitely generated $\mathcal{O}_{p}$-module.

(iii) If for all $l \in S$ the prime $p$ does not divide the ramification index of $l$ in $K_{\infty} / \mathbb{Q}$, then $\mathbf{R} \Gamma_{\mathrm{Iw}}\left(U_{K_{\infty}}, S, M(\varrho)\right)$ is a perfect torsion complex of $\mathcal{O}_{p} \llbracket \mathrm{G}\left(K_{\infty} / \mathbb{Q}\right) \rrbracket$-modules.

Supplement. If $K_{\infty} / \mathbb{Q}$ is a p-extension, $M=\mathcal{O}_{p}$, and $\varrho$ is ramified over a prime $l$ that is unramified in $K_{\infty} / \mathbb{Q}$, then

$$
\operatorname{char} \mathbf{R} \Gamma_{\mathrm{Iw}}\left(U_{K_{\infty}}, l, \mathcal{O}_{p}(\varrho)\right)=(1) .
$$

Proof. As in the proof of Proposition 4.2, we fix a number $m$ such that none of the primes in $S_{K_{m}}$ splits or ramifies in $K_{\infty} / K_{m}$.

Write $v_{m} \in S_{K_{m}}$ for the image of a point $v \in S_{K_{\infty}}$. From [Jan88, Prop. $3.8]$, we obtain an isomorphism of $\mathcal{O}_{p} \llbracket \mathrm{G}\left(K_{\infty} / K_{m}\right) \rrbracket$-modules

$$
\begin{aligned}
& \mathrm{H}_{\mathrm{Iw}}^{i}\left(U_{K_{\infty}}, S, M(\varrho)\right) \\
& \quad=\bigoplus_{v \in S_{K_{\infty}}} \mathbf{R}^{i}\left(\lim _{\longleftarrow} \Gamma_{\text {ét }}\right)\left(\operatorname{Spec} \mathcal{O}_{v_{m}}^{h}, v_{m}, j_{K_{m} *} \operatorname{Ind}_{K_{\infty} / K_{m}} M(\varrho)\right),
\end{aligned}
$$

where $\mathcal{O}_{v_{m}}^{h}$ is the henselisation of the local ring at $v_{m}$ and the limit is taken over the projective system $j_{K_{m} *} \operatorname{Ind}_{K_{\infty} / K_{m}} M(\varrho)$.

We will now use the connection between étale and Galois cohomology. Fix a $v \in S_{K_{\infty}}$ and let $G_{v_{m}}$ denote the absolute Galois group of $Q\left(\mathcal{O}_{v_{m}}^{h}\right)$, $I_{v_{m}}$ its inertia subgroup and $g_{v_{m}}=G_{v_{m}} / I_{v_{m}}$ the Galois group of the residue field of $v_{m}$. For any profinite group $G$, let $\operatorname{cd}_{p} G$ denote the cohomological 
$p$-dimension, i.e. the largest number $i$ such that the $i$ th group cohomology functor is non-trivial on finite $p$-torsion $G$-modules. We have

$$
\operatorname{cd}_{p} G_{v_{m}}=2, \quad \operatorname{cd}_{p} I_{v_{m}}=\operatorname{cd}_{p} g_{v_{m}}=1
$$

(see [NSW00, Prop. 3.3.4, Prop. 7.1.8]). Further, it is well known that for these groups, the cohomology groups of finite $p$-torsion modules will again be finite. In particular, we may interchange projective limits and continuous cohomology functors during the subsequent considerations.

By [Mil86, Proposition II.1.1(b)] and [Mil80, Ex. II.3.15] it follows that

$\mathbf{R}^{i}\left(\varliminf_{\longleftarrow} \Gamma_{\text {ét }}\right)\left(\operatorname{Spec} \mathcal{O}_{v_{m}}^{h}, j_{K_{m} *} \operatorname{Ind}_{K_{\infty} / K_{m}} M(\varrho)\right)$

$$
=\mathrm{H}^{i}\left(g_{v_{m}}, H^{0}\left(I_{v_{m}}, \operatorname{Ind}_{K_{\infty} / K_{m}} M(\varrho)\right)\right) .
$$

Comparing the localisation sequence for

$$
\operatorname{Spec} Q\left(\mathcal{O}_{v_{m}}^{h}\right) \hookrightarrow \operatorname{Spec} \mathcal{O}_{v_{m}}^{h} \hookleftarrow v_{m}
$$

with the Hochschild-Serre spectral sequence for $I_{v_{m}} \subset G_{v_{m}}$ we obtain from the above

$\mathbf{R}^{i}\left(\varliminf_{\longleftarrow} \Gamma_{\text {ét }}\right)\left(\operatorname{Spec} \mathcal{O}_{v_{m}}^{h}, v_{m}, j_{K_{m} *} \operatorname{Ind}_{K_{\infty} / K_{m}} M(\varrho)\right)$

$$
=\mathrm{H}^{i-2}\left(g_{v_{m}}, \mathrm{H}^{1}\left(I_{v_{m}}, \operatorname{Ind}_{K_{\infty} / K_{m}} M(\varrho)\right)\right) .
$$

As $v_{m}$ is unramified in $K_{\infty} / K_{m}$, the action of $I_{v_{m}}$ on $\mathcal{O}_{p} \llbracket \mathrm{G}\left(K_{\infty} / K_{m}\right) \rrbracket(\iota)$ is trivial, and hence,

$$
\mathrm{H}^{1}\left(I_{v_{m}}, \operatorname{Ind}_{K_{\infty} / K_{m}} M(\varrho)\right)=\operatorname{Ind}_{K_{\infty} / K_{m}} \mathrm{H}^{1}\left(I_{v_{m}}, M(\varrho)\right) .
$$

Observe that $\mathrm{H}^{1}\left(I_{v_{m}}, M(\varrho)\right)$ is a finitely generated $\mathcal{O}_{p}$-module.

To finish the proof of parts (i) and (ii) of Proposition 5.1 it remains to verify the following

Lemma 5.2. Let $N(\tau)$ be a finitely generated $\mathcal{O}_{p}$-module $N$ together with a continuous representation $\tau: g_{v_{m}} \rightarrow \operatorname{Aut}_{\mathcal{O}_{p}} N$. Then

(i) $\mathrm{H}^{0}\left(g_{v_{m}}, \operatorname{Ind}_{K_{\infty} / K_{m}} N(\tau)\right)=0$,

(ii) $\mathrm{H}^{1}\left(g_{v_{m}}, \operatorname{Ind}_{K_{\infty} / K_{m}} N(\tau)\right)$ is a finitely generated $\mathcal{O}_{p}$-module.

Proof. By our assumption on $m$ we have $I_{v_{n}}=I_{v_{m}}$ and $G_{v_{n}} / G_{v_{m}}=$ $\mathrm{G}\left(K_{n} / K_{m}\right)$ for $n \geq m$. Thus,

$$
\mathrm{H}^{i}\left(g_{v_{m}}, \operatorname{Ind}_{K_{\infty} / K_{m}} N(\tau)\right)=\lim _{n} \mathrm{H}^{i}\left(g_{v_{n}}, N(\tau)\right) .
$$

The same argument as in Proposition 3.3(ii) implies that this term vanishes for $i=0$. This proves (i). Claim (ii) follows because $\mathrm{H}^{1}\left(g_{v_{m}}, N(\tau)\right)$ is a quotient of $N$ for all $n$.

We now prove Proposition 5.1(iii). After decomposing by characters we may assume that $K_{\infty}$ is a $p$-extension. In particular, $l \in S$ is unramified. By 
the same argument as above, replacing $K_{m}$ by $\mathbb{Q}$, we obtain

$$
\mathrm{H}_{\mathrm{Iw}}^{3}\left(U_{K_{\infty}}, l, M(\varrho)\right)=\mathrm{H}^{1}\left(g_{l}, \mathrm{H}^{1}\left(I_{l}, \operatorname{Ind}_{K_{\infty} / \mathbb{Q}} M(\varrho)\right)\right) .
$$

Write $\Omega=\mathcal{O}_{p} \llbracket \mathrm{G}\left(K_{\infty} / \mathbb{Q}\right) \rrbracket$ and note that $I_{l}$ acts trivially on $\Omega(\iota)$. Consequently,

$$
\mathrm{H}^{1}\left(I_{l}, \operatorname{Ind}_{K_{\infty} / \mathbb{Q}} M(\varrho)\right)=\operatorname{Ind}_{K_{\infty} / \mathbb{Q}} N(\tau),
$$

where $N=\mathrm{H}^{1}\left(I_{l}, M(\varrho)\right)$ is a finitely generated $\mathcal{O}_{p}$-module and $\tau: g_{l} \rightarrow$ Aut $_{\mathcal{O}_{p}} N$ is the induced representation. Recall that $g_{l}$ is topologically generated by the geometric Frobenius element $\mathcal{F}_{l}$. By (i) the sequence

$$
0 \rightarrow \Omega \otimes_{\mathcal{O}_{p}} N \stackrel{\text { id }-\iota\left(\mathcal{F}_{l}\right) \otimes \tau\left(\mathcal{F}_{l}\right)}{\longrightarrow} \Omega \otimes_{\mathcal{O}_{p}} N \rightarrow \mathrm{H}_{\mathrm{Iw}}^{3}\left(U_{K_{\infty}}, l, M(\varrho)\right) \rightarrow 0
$$

is exact. As $\mathcal{O}_{p}$ is regular, $\Omega \otimes_{\mathcal{O}_{p}} N$ is perfect as $\Omega$-complex; hence, so is $\mathrm{H}_{\mathrm{Iw}}^{3}\left(U_{K_{\infty}}, l, M(\varrho)\right)$. The latter module is $\Omega$-torsion, because it is a finitely generated $\mathcal{O}_{p}$-module. This proves (iii).

To prove the Supplement it suffices to recall that

$$
N=\left(M(\varrho)^{R_{l}}\right)_{T_{l}},
$$

where $R_{l}$ is the ramification subgroup and $T_{l}=I_{l} / R_{l}$. If $M=\mathcal{O}_{p}$ and the restriction of $\varrho$ to $I_{l}$ is non-trivial, then this module is clearly $\mathcal{O}_{p}$-torsion. By parts (iii) and (v) of Proposition 1.5 applied to the above exact sequence, we obtain

$$
\operatorname{char} \mathbf{R} \Gamma_{\mathrm{Iw}}\left(U_{K_{\infty}}, l, M(\varrho)\right)=\operatorname{char}\left(\Omega \otimes_{\mathcal{O}_{p}} N\right) \operatorname{char}^{-1}\left(\Omega \otimes_{\mathcal{O}_{p}} N\right)=(1) .
$$

This finishes the proof of Proposition 5.1.

REMARK 5.3. Let $W$ be a finitely generated $\Omega$-module. If either $W$ or $\mathrm{H}^{1}\left(I_{l}, M(\varrho)\right)$ is flat as an $\mathcal{O}_{p}$-module, then

$$
\begin{aligned}
W \otimes \mathbb{\Omega}_{\Omega}^{\mathbb{L}} \mathbf{R} \Gamma_{\mathrm{Iw}}\left(U_{K_{\infty}}, l, \operatorname{Ind}_{K_{\infty} / \mathbb{Q}}\right. & M(\varrho)) \\
& \cong \mathrm{H}^{1}\left(g_{l}, W \otimes_{\Omega} \operatorname{Ind}_{K_{\infty} / \mathbb{Q}} \mathrm{H}^{1}\left(I_{l}, M(\varrho)\right)\right) \\
& \cong \mathbf{R} \Gamma_{\mathrm{Iw}}\left(U_{K_{\infty}}, l, W \otimes_{\Omega} \operatorname{Ind}_{K_{\infty} / \mathbb{Q}} M(\varrho)\right) .
\end{aligned}
$$

This is not true without the additional flatness assumption.

6. Cyclotomic elements and $L$-elements. The aim of this section is to assign to each admissible triple $\left(K_{\infty}, \varrho, U\right)$ given by

- a cyclotomic $\mathbb{Z}_{p}$-extension $K_{\infty}$ of an abelian number field,

- a representation $\varrho: \mathrm{G}(\overline{\mathbb{Q}} / \mathbb{Q}) \rightarrow \mathcal{O}_{p}^{\times}$,

- an open subscheme $U$ of Spec $\mathbb{Z}$ that does not contain any prime whose ramification index in $K_{\infty} / \mathbb{Q}$ is divisible by $p$

an $L$-element $L\left(U_{K_{\infty}}, \varrho^{-1} \varepsilon_{\text {cycl }}\right) \in Q\left(\mathcal{O}_{p} \llbracket \mathrm{G}\left(K_{\infty} / \mathbb{Q}\right) \rrbracket\right)^{\times}$and a cyclotomic element $c\left(U_{K_{\infty}}, \varrho\right) \in \mathrm{H}_{\mathrm{Iw}}^{1}\left(U_{K_{\infty}}, \mathcal{O}_{p}(\varrho)\right)$. 
If $\varrho$ and $U_{K_{\infty}}$ are unramified over $U$, then our definition follows along the lines of the classical construction (see [Was97, $\S 7.2]$, respectively [Rub00, $\S 3.2]$ ). By the theorem of Kronecker and Weber there exists a number $f$ such that

- the set of prime divisors of $f$ is equal to the complement of $U$ in Spec $\mathbb{Z}[1 / p]$,

- $K_{\infty} \subset \mathbb{Q}\left(\zeta_{f p^{\infty}}\right)$

- $\varrho$ factors through $\mathrm{G}\left(\mathbb{Q}\left(\zeta_{f p^{\infty}}\right) / \mathbb{Q}\right)$.

Let $\mathcal{F}_{l}$ denote the geometric Frobenius element and set

$$
\mathcal{F}_{a}=\prod_{l \text { prime }} \mathcal{F}_{l}^{v_{l}(a)}
$$

for each positive integer $a$. The Stickelberger elements

$$
\xi_{f p^{k}}=\sum_{\substack{0<a<f p^{k} \\(a, f p)=1}}\left(\frac{a}{f p^{k}}-\frac{1}{2}\right) \mathcal{F}_{a} \in \mathbb{Q}_{p}\left[\mathrm{G}\left(\mathbb{Q}\left(\zeta_{f p^{k}}\right) / \mathbb{Q}\right)\right]
$$

are compatible under the projection maps induced by

$$
\mathrm{G}\left(\mathbb{Q}\left(\zeta_{f p^{k+1}}\right) / \mathbb{Q}\right) \rightarrow \mathrm{G}\left(\mathbb{Q}\left(\zeta_{f p^{k}}\right) / \mathbb{Q}\right)
$$

and define an element

$$
\xi_{f p^{\infty}} \in Q\left(\mathcal{O}_{p} \llbracket \mathrm{G}\left(\mathbb{Q}\left(\zeta_{f p^{\infty}}\right) / \mathbb{Q}\right) \rrbracket\right) .
$$

Further, we fix for each number $k$ a primitive $k$ th root of unity $\zeta_{k}$ such that $\zeta_{k s}^{s}=\zeta_{k}$. By Proposition 4.1 we may regard the system $c_{f p^{\infty}}=\left(1-\zeta_{f p^{k}}\right)_{k=0}^{\infty}$ as an element of $\mathrm{H}_{\mathrm{Iw}}^{1}\left(U_{\mathbb{Q}\left(\zeta_{f p} \infty\right)}, \mathcal{O}_{p}\left(\varepsilon_{\text {cycl }}\right)\right)$.

Definition 6.1. Let $\varrho$ and $U_{K_{\infty}}$ be unramified over $U$. Denote by $p_{+}$and $p_{-}$the projectors onto the $(+1)$-eigenspace, respectively the $(-1)$-eigenspace of the complex conjugation and chose $f$ as above. We set

$$
L\left(U_{K_{\infty}}, \varrho^{-1} \varepsilon_{\text {cycl }}\right)=\psi_{\mathbb{Q}\left(\zeta_{f p} \infty\right) / K_{\infty}} \operatorname{Tw}_{\varrho^{-1} \varepsilon_{\text {cycl }}}\left(p_{+}-p_{-} \xi_{f p^{\infty}}\right) .
$$

The cyclotomic element $c\left(U_{K_{\infty}}, \varrho\right)$ is defined to be the image of $1 \otimes p_{+} c_{f p^{\infty}}$ under the homomorphism

$$
\left(\psi_{\mathbb{Q}\left(\zeta_{f p} \infty\right) / K_{\infty}} \mathrm{Tw}_{\varrho^{-1} \varepsilon_{\mathrm{cycl}}}\right)_{*} \mathrm{H}_{\mathrm{Iw}}^{1}\left(U_{\mathbb{Q}\left(\zeta_{f p} \infty\right)}, \mathcal{O}_{p}\left(\varepsilon_{\mathrm{cycl}}\right)\right) \rightarrow \mathrm{H}_{\mathrm{Iw}}^{1}\left(U_{K_{\infty}}, \mathcal{O}_{p}(\varrho)\right) .
$$

For any $l \in U$ we denote by

$$
E_{l}\left(K_{\infty}, \varrho^{-1} \varepsilon_{\text {cycl }}\right)=1-\psi_{\mathbb{Q}\left(\zeta_{f p} \infty\right) / K_{\infty}} \operatorname{Tw}_{\varrho^{-1} \varepsilon_{\text {cycl }}}\left(\mathcal{F}_{l}\right)
$$

the Euler factor at $l$.

We now extend this definition to arbitrary admissible triples $\left(K_{\infty}, \varrho, U\right)$. Let $P_{\infty}$ be the maximal $p$-extension inside $K_{\infty}$. We may decompose the group ring $\mathcal{O}_{p} \llbracket \mathrm{G}\left(K_{\infty} / \mathbb{Q}\right) \rrbracket$ by the characters of $\mathrm{G}\left(K_{\infty} / P_{\infty}\right)$. The $L$-elements and cyclotomic elements for $K_{\infty}$ are then completely determined by their 
projections onto the components of the corresponding decomposition of $Q\left(\mathcal{O}_{p} \llbracket \mathrm{G}\left(K_{\infty} / \mathbb{Q}\right) \rrbracket\right)^{\times}$, respectively $\mathrm{H}_{\mathrm{Iw}}^{1}\left(U_{K_{\infty}}, \mathcal{O}_{p}(\varrho)\right)$. Consequently, it suffices to consider triples $\left(P_{\infty}, \varrho, U\right)$ with $P_{\infty}$ a $p$-extension. Note that every ramification index is now a power of $p$. By assumption, $U_{P_{\infty}}$ is therefore an unramified cover of $U$. In other words, we only need to deal with the ramification of $\varrho$.

Let $V \subset U$ be the maximal open subscheme such that $\varrho$ is unramified over $V$ (note that the complement is a finite set because $\varrho$ is one-dimensional) and set

$$
L\left(U_{P_{\infty}}, \varrho^{-1} \varepsilon_{\text {cycl }}\right)=L\left(V_{P_{\infty}}, \varrho^{-1} \varepsilon_{\text {cycl }}\right), \quad c\left(U_{P_{\infty}}, \varrho\right)=c\left(V_{P_{\infty}}, \varrho\right),
$$

and for any $l \in U-V$,

$$
E_{l}\left(P_{\infty}, \varrho^{-1} \varepsilon_{\text {cycl }}\right)=1 .
$$

It is easy to check that this definition is consistent with the previous construction. Moreover, we have

Proposition 6.2. Let $\left(K_{\infty}, U, \varrho\right)$ be any admissible triple.

(i) The elements $L\left(U_{K_{\infty}}, \varrho^{-1} \varepsilon_{\text {cycl }}\right), c\left(U_{K_{\infty}}, \varrho\right)$, and $E_{l}\left(K_{\infty}, \varrho^{-1} \varepsilon_{\text {cycl }}\right)$ are compatible under the projection maps $\psi_{K_{\infty} / L_{\infty}}$ and under twists by continuous characters $\mathrm{G}\left(K_{\infty} / \mathbb{Q}\right) \rightarrow \mathcal{O}_{p}^{\times}$.

(ii) Let $V \subset U$ be an open subscheme with closed complement $T=U-V$. Then

$$
\begin{aligned}
& L\left(V_{K_{\infty}}, \varrho\right)=\operatorname{Tw}_{\varrho}\left(p_{+}\right)+\operatorname{Tw}_{\varrho}\left(p_{-}\right) L\left(U_{K_{\infty}}, \varrho\right) \prod_{l \in T} E_{l}\left(K_{\infty}, \varrho\right), \\
& c\left(V_{K_{\infty}}, \varrho\right)=c\left(U_{K_{\infty}}, \varrho\right) \prod_{l \in T} E_{l}\left(K_{\infty}, \varrho^{-1} \varepsilon_{\text {cycl }}\right) .
\end{aligned}
$$

Proof. This is partly implied by the corresponding compatibility properties of $\xi_{f p^{\infty}}$ and $c_{f p^{\infty}}$, respectively, and partly a direct consequence of the definition.

We want to sketch briefly the connection between our $L$-elements and the Kubota-Leopoldt $L$-function $L_{p}(s, \chi)$. Let $K_{\infty}=\mathbb{Q}_{\infty}$ and $U=\operatorname{Spec} \mathbb{Z}[1 / p]$. According to the decomposition $\mathcal{O}_{p}^{\times}=\mu \times F$ into the torsion group of roots of unity $\mu$ and the torsion-free $\mathbb{Z}_{p}$-module $F$ we can write

$$
\varrho=\varrho_{f} \varrho_{\infty},
$$

where $\varrho_{f}$ takes values in $\mu$ and

$$
\varrho_{\infty}: \mathrm{G}\left(\mathbb{Q}_{\infty} / \mathbb{Q}\right) \rightarrow F
$$

is a continuous group homomorphism.

Let $N$ be the conductor of $\varrho_{f}$ and

$$
\text { rec }: \mathrm{G}\left(\mathbb{Q}\left(\zeta_{N}\right) / \mathbb{Q}\right) \rightarrow(\mathbb{Z} / N \mathbb{Z})^{\times}
$$


the isomorphism which maps the geometric Frobenius $\mathcal{F}_{l}$ to $l$, when $l$ is prime to $N$. Further, note that the number

$$
s=\frac{\log _{p} \varrho_{\infty}(\gamma)}{\log _{p}\left(\left(\varepsilon_{\mathrm{cycl}}\right)_{\infty}(\gamma)\right)} \in \log _{p} F \subset \mathbb{C}_{p}
$$

does not depend on the choice of a non-trivial $\gamma \in \mathrm{G}\left(\mathbb{Q}_{\infty} / \mathbb{Q}\right)$. Hence, we may write $\varrho_{\infty}=\left(\varepsilon_{\text {cycl }}\right)_{\infty}^{s}$.

Proposition 6.3. Let $\varrho=\left(\varepsilon_{\mathrm{cycl}}\right)_{\infty}^{s} \varrho_{f}$ be an even one-dimensional representation. Then

$$
\psi_{\mathbb{Q}_{\infty} / \mathbb{Q}}\left(L\left(U_{\mathbb{Q}_{\infty}}, \varepsilon_{\mathrm{cycl}} \varrho\right)\right)=L_{p}\left(1+s, \varrho_{f} \circ \mathrm{rec}^{-1}\right) .
$$

Proof. See [Was97, Theorem 7.10], but observe that the identification

$$
\mathrm{G}\left(\mathbb{Q}\left(\zeta_{N}\right) / \mathbb{Q}\right) \rightarrow(\mathbb{Z} / N \mathbb{Z})^{\times}
$$

used in loc. cit. is given by $1 /$ rec. In particular, $w \circ$ rec $=\left(\varepsilon_{\text {cycl }}\right)_{f}^{-1}$, where $w$ denotes the Teichmüller character.

REMARK 6.4. Let $N$ be prime to $p$ and let $\chi:(\mathbb{Z} / N p \mathbb{Z})^{\times} \rightarrow \mathcal{O}_{p}^{\times}$be an odd Dirichlet character of conductor $N$ or $N p$ (i.e. $\chi$ is of the first kind). Set $U=\operatorname{Spec} \mathbb{Z}[1 / p]$ and let

$$
\beta: \mathcal{O}_{p} \llbracket \mathrm{G}\left(\mathbb{Q}_{\infty} / \mathbb{Q}\right) \rrbracket \rightarrow \mathcal{O}_{p} \llbracket T \rrbracket
$$

be the isomorphism that maps $\mathcal{F}_{1+N p}^{-1}$ to $T+1$. By construction we then have

$$
f(T, \chi \omega)=\beta\left(L\left(U_{\mathbb{Q}_{\infty}}, \chi \circ \text { rec }\right)\right),
$$

where $f(T, \chi \omega)$ is the element introduced in [Was97, §7.2].

Remark 6.5. Let $\chi$ be a finite character and $k$ an integer. Proposition 6.3 implies that our element $L\left(U_{\mathbb{Q}_{\infty}}, \varepsilon_{\text {cycl }}^{1-k} \chi\right)$, with $U=\operatorname{Spec} \mathbb{Z}[1 / p]$, coincides with the $p$-adic $L$-function $\mathcal{L}_{p}(\chi, 1-k)$ used in [HK03]. However, note that there is a sign error in the definition of this function. The correct definition should read as follows (in the notation of loc. cit.). For all $\widetilde{\mathcal{O}}_{p}$ and all characters $\tau: \Gamma \rightarrow \widetilde{\mathcal{O}}_{p}^{*}$ of finite order,

$$
\tau\left(\mathcal{L}_{p}(\chi, 1-k)\right)=\left(1-\chi \tau(p) p^{k-1}\right) L(\chi \tau, 1-k) .
$$

REMARK 6.6. The elements $c\left(U_{K_{\infty}}, \varrho\right)$ depend on the choice of the system of roots of unity $\left(\zeta_{k}\right)$, but the submodule of $\mathrm{H}_{\mathrm{Iw}}^{1}\left(U_{K_{\infty}}, \mathcal{O}_{p}(\varrho)\right)$ generated by $c\left(U_{K_{\infty}}, \varrho\right)$ does not. This is the actual object we are interested in.

The theorem of Ferrero-Washington can be rephrased to the statement that the $\mu$-invariants of the $p$-adic $L$-functions vanish. The following proposition translates this formulation to our setting.

Proposition 6.7. Let $\left(K_{\infty}, U, \varrho\right)$ be an admissible triple and $l \in \operatorname{Spec} \mathbb{Z}$ of prime-to-p ramification in $K_{\infty} / \mathbb{Q}$. Then $L\left(U_{K_{\infty}}, \varrho\right)$ and $E_{l}\left(K_{\infty}, \varrho\right)$ map 
to units in $\mathcal{O}_{p} \llbracket \mathrm{G}\left(K_{\infty} / \mathbb{Q}\right) \rrbracket_{\mathfrak{p}}$ for each prime ideal $\mathfrak{p}$ of codimension 1 with $p \in \mathfrak{p}$.

Proof. By Proposition 6.2(ii) we may assume that $U_{K_{\infty}}$ and $\varrho$ are unramified over $U$. Choose $f$ as above and observe that

$$
\left(1-(1+f p) \mathcal{F}_{1+f p}\right) \xi_{f p^{\infty}} \in \mathcal{O}_{p} \llbracket \mathrm{G}\left(\mathbb{Q}\left(\zeta_{f p^{\infty}}\right) / \mathbb{Q}\right) \rrbracket .
$$

Define

$$
h\left(U_{K_{\infty}}, \varrho\right)=1-(1+f p) \psi_{\mathbb{Q}\left(\zeta_{f p} \infty\right) / K_{\infty}} \operatorname{Tw}_{\varrho}\left(\mathcal{F}_{1+f p}\right) .
$$

We need to show that none of $h\left(U_{K_{\infty}}, \varrho\right), h L\left(U_{K_{\infty}}, \varrho\right)$, or $E_{l}\left(K_{\infty}, \varrho\right)$ is contained in $\mathfrak{p}$. For this, we can replace $\mathcal{O}_{p} \llbracket \mathrm{G}\left(K_{\infty} / \mathbb{Q}\right) \rrbracket$ by its normalisation and then decompose by the characters of $\mathrm{G}\left(K_{0} / \mathbb{Q}\right)$. Hence, we may assume that $K_{\infty}=\mathbb{Q}_{\infty}$. In particular, $\mathfrak{p}$ is the radical of $(p)$. After twisting by an appropriate character of $\mathrm{G}\left(\mathbb{Q}_{\infty} / \mathbb{Q}\right)$ we may further require that $\varrho=\chi \circ \operatorname{rec}^{-1}$ for a Dirichlet character $\chi$ of the first kind.

Obviously, $E_{l}\left(\mathbb{Q}_{\infty}, \chi\right)$ and $h\left(U_{\mathbb{Q}_{\infty}}, \chi\right)$ are prime to $p$ (note that the images of $\mathcal{F}_{l}$ and $\mathcal{F}_{1+f p}$ are non-trivial in $\left.\mathrm{G}\left(\mathbb{Q}_{\infty} / \mathbb{Q}\right)\right)$. If $\chi$ is even, then $L\left(U_{K_{\infty}}, \chi\right)$ $=1$. If $\chi$ is odd, the claim for $h L\left(U_{K_{\infty}}, \theta\right)$ is by Remark 6.4 equivalent to the vanishing of the $\mu$-invariant of $f(T, \chi \omega)$, hence to the theorem of Ferrero-Washington ([Was97, §7.5, respectively $§ 16.2]$ ).

7. The main theorem. Let $\left(K_{\infty}, \varrho, U\right)$ be an admissible triple in the sense of Section 6 and set

$$
\Omega=\mathcal{O}_{p} \llbracket \mathrm{G}\left(K_{\infty} / \mathbb{Q}\right) \rrbracket,
$$

where $\mathcal{O}_{p}$ is the valuation ring of a finite extension of $\mathbb{Q}_{p}$. As explained in Section 2, we may assume without loss of generality that $\mathcal{O}_{p}$ contains the values of all characters of $\mathrm{G}\left(K_{0} / \mathbb{Q}\right)$.

Before we state our main theorem we will explain how to modify the Iwasawa complex $\mathbf{R} \Gamma_{\mathrm{Iw}}\left(U_{K_{\infty}}, \mathcal{O}_{p}(\varrho)\right.$ ) (see Definition 3.1 ) by the cyclotomic element

$$
c\left(U_{K_{\infty}}, \varrho\right) \in \mathrm{H}_{\mathrm{Iw}}^{1}\left(U_{K_{\infty}}, \mathcal{O}_{p}(\varrho)\right)
$$

introduced in the preceding section.

Recall that the complex $\mathbf{R} \Gamma_{\text {Iw }}\left(U_{K_{\infty}}, \mathcal{O}_{p}(\varrho)\right)$ is acyclic in degree 0 (see Proposition 3.3(ii)). In particular, there exists a unique morphism

$$
\Omega c\left(U_{K_{\infty}}, \varrho\right)[-1] \rightarrow \mathbf{R} \Gamma_{\mathrm{Iw}}\left(U_{K_{\infty}}, \mathcal{O}_{p}(\varrho)\right)
$$

in the derived category that induces the natural inclusion on cohomology.

Definition 7.1. Denote by $\mathbf{R} \Gamma_{\text {Iw }} / c\left(U_{K_{\infty}}, \mathcal{O}_{p}(\varrho)\right)$ the complex (unique up to quasi-isomorphism) fitting into the following distinguished triangle:

$$
\Omega c\left(U_{K_{\infty}}, \varrho\right)[-1] \rightarrow \mathbf{R} \Gamma_{\mathrm{Iw}}\left(U_{K_{\infty}}, \mathcal{O}_{p}(\varrho)\right) \rightarrow \mathbf{R} \Gamma_{\mathrm{Iw}} / c\left(U_{K_{\infty}}, \mathcal{O}_{p}(\varrho)\right)
$$


LEMMA 7.2.

(i) $\mathbf{R} \Gamma_{\mathrm{Iw}} / c\left(U_{K_{\infty}}, \mathcal{O}_{p}(\varrho)\right)$ is a perfect torsion complex of $\Omega$-modules.

(ii) If $K_{\infty}$ is totally real and $\varrho$ is odd, then $\Omega c\left(U_{K_{\infty}}, \varrho\right)$ is a free $\Omega$ module of rank 1 .

Proof. In Proposition 3.6(i) we have already confirmed that the complex $\mathbf{R} \Gamma_{\text {Iw }}\left(U_{K_{\infty}}, \mathcal{O}_{p}(\varrho)\right)$ is perfect. Hence, it suffices to prove (ii) and that the complex in (i) is torsion.

Let $\widetilde{\Omega}$ denote the normalisation of $\Omega$ in its total quotient $\operatorname{ring} Q(\Omega)$. On the one hand we have

$$
Q(\Omega) \otimes_{\Omega} \mathrm{H}_{\mathrm{Iw}}^{i}\left(U_{K_{\infty}}, \mathcal{O}_{p}(\varrho)\right)=Q(\Omega) \otimes_{\widetilde{\Omega}}\left(\bigoplus_{\chi} \mathrm{H}_{\mathrm{Iw}}^{i}\left(U_{\mathbb{Q} \infty}, \mathcal{O}_{p}\left(\chi^{-1} \varrho\right)\right)\right),
$$

where the sum runs over all characters $\chi$ of $\mathrm{G}\left(K_{0} / \mathbb{Q}\right)$; on the other hand $\Omega c\left(U_{K_{\infty}}, \varrho\right) \subset \mathrm{H}_{\mathrm{Iw}}^{1}\left(U_{K_{\infty}}, \mathcal{O}_{p}(\varrho)\right)$ is a free $\Omega$-module of rank 1 if and only if $Q(\Omega) c\left(U_{K_{\infty}}, \varrho\right)$ is a free $Q(\Omega)$-module of rank 1 . This module decomposes as

$$
Q(\Omega) c\left(U_{K_{\infty}}, \varrho\right)=\bigoplus_{\chi} Q\left(\mathcal{O}_{p} \llbracket \mathrm{G}\left(\mathbb{Q}_{\infty} / \mathbb{Q}\right) \rrbracket\right) c\left(U_{\mathbb{Q} \infty}, \chi^{-1} \varrho\right) .
$$

Observe hereby that $\varrho$ and $\chi^{-1} \varrho$ have the same parity if $K_{\infty}$ is totally real. Hence, it is enough to consider the case $K_{\infty}=\mathbb{Q}_{\infty}$. By Propositions 5.1 and 6.2 we may replace $U$ by $\operatorname{Spec} \mathbb{Z}[1 / p]$, noting that the Euler factors $E_{l}\left(\mathbb{Q}_{\infty}, \varrho\right)$ are non-zero divisors. We are now reduced to the statement of [HK03, Proposition 4.2.1]. Observe that the proof of (ii) in this situation uses K. Kato's explicit reciprocity law as an essential ingredient.

Corollary 7.3. The compatibility properties of Proposition 3.6 hold for $\mathcal{O}_{p}(\varrho)$, with $\mathbf{R} \Gamma_{\text {Iw }}$ replaced by $\mathbf{R} \Gamma_{\text {Iw }} / c$.

Proof. Easy consequence of Proposition 6.2 and the above lemma.

We are now ready to formulate and prove our main result.

THEOREM 7.4. Let $p$ be an odd prime, $K_{\infty}$ the cyclotomic $\mathbb{Z}_{p}$-extension of an abelian number field, and $U$ an open subscheme of Spec $\mathbb{Z}$ such that the ramification index in $K_{\infty} / \mathbb{Q}$ of every place in $U$ is prime to $p$. Then:

(i) $\mathbf{R} \Gamma_{\mathrm{Iw}} / c\left(U_{K_{\infty}}, \mathcal{O}_{p}(\varrho)\right)_{\mathfrak{p}}$ is acyclic for all primes $\mathfrak{p}$ of codimension 1 of $\mathcal{O}_{p} \llbracket \mathrm{G}\left(K_{\infty} / \mathbb{Q}\right) \rrbracket$ that contain $p$.

(ii) The characteristic ideal of $\mathbf{R} \Gamma_{\mathrm{Iw}} / c\left(U_{K_{\infty}}, \mathcal{O}_{p}(\varrho)\right)$ is generated by the $L$-element $L\left(U_{K_{\infty}}, \varrho^{-1} \varepsilon_{\text {cycl }}\right)$.

Proof. By the subsequent lemma we are allowed to enlarge or shrink the scheme $U$ at our discretion.

LEMMA 7.5. Let $V \subset U$ be an open subscheme of $U$. Then both statements of Theorem 7.4 hold for $U$ if and only if they hold for $V$. 
Proof. Set $T=U-V$ and define $C^{\bullet}$ by the following distinguished triangle:

$$
\Omega c\left(V_{K_{\infty}}, \varrho\right)[-1] \rightarrow \mathbf{R} \Gamma_{\mathrm{Iw}}\left(U_{K_{\infty}}, \mathcal{O}_{p}(\varrho)\right) \rightarrow C^{\bullet},
$$

where the first map is induced by the inclusion

$$
\Omega c\left(V_{K_{\infty}}, \varrho\right) \hookrightarrow \mathrm{H}_{\mathrm{Iw}}^{1}\left(V_{K_{\infty}}, \mathcal{O}_{p}(\varrho)\right)=\mathrm{H}_{\mathrm{Iw}}^{1}\left(U_{K_{\infty}}, \mathcal{O}_{p}(\varrho)\right) .
$$

We obtain the following two triangles of perfect torsion complexes:

$$
\begin{aligned}
& \mathbf{R} \Gamma_{\mathrm{Iw}}\left(U_{K_{\infty}}, T, \mathcal{O}_{p}(\varrho)\right) \rightarrow C \bullet \rightarrow \mathbf{R} \Gamma_{\mathrm{Iw}} / c\left(V_{K_{\infty}}, \mathcal{O}_{p}(\varrho)\right), \\
& C^{\bullet} \rightarrow \mathbf{R} \Gamma_{\mathrm{Iw}} / c\left(U_{K_{\infty}}, \mathcal{O}_{p}(\varrho)\right) \rightarrow \Omega c\left(U_{K_{\infty}}, \varrho\right) / \Omega c\left(V_{K_{\infty}}, \varrho\right) .
\end{aligned}
$$

Proposition 6.2 implies

$$
\Omega c\left(U_{K_{\infty}}, \varrho\right) / \Omega c\left(V_{K_{\infty}}, \varrho\right)=\operatorname{Tw}_{\varrho^{-1} \varepsilon_{\mathrm{cycl}}}\left(p_{+}\right)\left(\Omega / \prod_{l \in T} E_{l}\left(K_{\infty}, \varrho^{-1} \varepsilon_{\mathrm{cycl}}\right) \Omega\right)
$$

and since $E_{l}\left(K_{\infty}, \varrho^{-1} \varepsilon_{\text {cycl }}\right)$ is a unit of $\Omega_{\mathfrak{p}}$ for any prime ideal $\mathfrak{p}$ of codimension 1 with $p \in \mathfrak{p}$ (see Proposition 6.7) it follows that

$$
\left(\Omega c\left(U_{K_{\infty}}, \varrho\right) / \Omega c\left(V_{K_{\infty}}, \varrho\right)\right)_{\mathfrak{p}}=0 .
$$

On the other hand, we know that $\mathbf{R} \Gamma_{\mathrm{Iw}}\left(U_{K_{\infty}}, T, \mathcal{O}_{p}(\varrho)\right)$ is acyclic outside degree 3 and that $\mathrm{H}_{\mathrm{Iw}}^{3}\left(U_{K_{\infty}}, T, \mathcal{O}_{p}(\varrho)\right)$ is finitely generated as $\mathcal{O}_{p}$-module (see Proposition 5.1). Therefore,

$$
\mathbf{R} \Gamma_{\mathrm{Iw}}\left(U_{K_{\infty}}, T, \mathcal{O}_{p}(\varrho)\right)_{\mathfrak{p}} \cong 0
$$

by Lemma 2.2. This implies the equivalence for part (i) of Theorem 7.4.

By using the multiplicativity of the characteristic ideal, the equivalence for part (ii) is reduced to proving that

$$
\operatorname{char} \mathrm{H}_{\mathrm{Iw}}^{3}\left(U_{K_{\infty}}, l, \mathcal{O}_{p}(\varrho)\right)=E_{l}\left(K_{\infty}, \varrho^{-1} \varepsilon_{\text {cycl }}\right) \Omega
$$

for $l \in T$. After decomposing by characters we may assume that $K_{\infty}$ is a $p$-extension. For those primes over which $\varrho$ is ramified the equality is implied by the Supplement in Proposition 5.1. For the remaining primes choose $f$ as in Section 6. From Corollary 4.3 we obtain

$$
\begin{aligned}
\operatorname{char} \mathrm{H}_{\mathrm{Iw}}^{3}\left(U_{K_{\infty}}, l, \mathcal{O}_{p}(\varrho)\right) \\
=\operatorname{char}\left(\psi_{\mathbb{Q}\left(\zeta_{f p} \infty\right) / K_{\infty}} \operatorname{Tw}_{\varrho^{-1} \varepsilon_{\mathrm{cycl}}}\right)_{*} \mathcal{O}_{p}\left[\mathrm{G}\left(\mathbb{Q}\left(\zeta_{f p^{\infty}}\right) / \mathbb{Q}\right) / D_{l}\right] \\
=E_{l}\left(K_{\infty}, \varrho^{-1} \varepsilon_{\mathrm{cycl}}\right) \Omega .
\end{aligned}
$$

From the formulation of the main conjecture in [HK03] we can deduce the following weaker instance of Theorem 7.4(ii).

Lemma 7.6. Let $\phi: \Omega \rightarrow \widetilde{\Omega}$ be the normalisation of $\Omega$. Then $\operatorname{char} \mathbf{L} \phi_{*} \mathbf{R} \Gamma_{\mathrm{Iw}} / c\left(U_{K_{\infty}}, \mathcal{O}_{p}(\varrho)\right)=L\left(U_{K_{\infty}}, \varrho^{-1} \varepsilon_{\mathrm{cycl}}\right) \widetilde{\Omega}$. 
Proof. We may decompose by the characters of $\mathrm{G}\left(K_{0} / \mathbb{Q}\right)$. Thus, we may assume that $K_{\infty}=\mathbb{Q}_{\infty}$. By Lemma 7.5 we can further reduce to $U=$ $\operatorname{Spec} \mathbb{Z}[1 / p]$.

If $\varrho$ is odd, then $L\left(U_{\mathbb{Q}_{\infty}}, \varrho^{-1} \varepsilon_{\text {cycl }}\right)=1$ by definition. On the other hand,

$$
\operatorname{char} \mathbf{R} \Gamma_{\mathrm{Iw}} / c\left(U_{\mathbb{Q}_{\infty}}, \mathcal{O}_{p}(\varrho)\right)=(1)
$$

by [HK03, Theorem 4.2.2]. If $\varrho$ is even, then $c\left(U_{\mathbb{Q}_{\infty}}, \varrho\right)=0$ and, by [HK03, Theorem 4.2.4],

$$
\operatorname{char} \mathbf{R} \Gamma_{\mathrm{Iw}}\left(U_{\mathbb{Q}_{\infty}}, \mathcal{O}_{p}(\varrho)\right)=L\left(U_{\mathbb{Q}_{\infty}}, \varrho^{-1} \varepsilon_{\text {cycl }}\right) \mathcal{O}_{p} \llbracket \mathrm{G}\left(\mathbb{Q}_{\infty} / \mathbb{Q}\right) \rrbracket .
$$

Originally, both theorems only deal with the case that $\varrho$ is a finite character times an integral power of $\varepsilon_{\text {cycl }}$, but the general case follows easily by twisting.

We now turn to the proof of Theorem 7.4(i). A large portion of it can be dealt with by the following lemma. Here, we use the theorem of FerreroWashington for the second time (see Proposition 6.7).

Lemma 7.7. $\mathrm{Tw}_{\varrho^{-1} \varepsilon_{\text {cycl }}}\left(p_{-}\right) \mathrm{H}_{\mathrm{Iw}}^{1}\left(U_{K_{\infty}}, \mathcal{O}_{p}(\varrho)\right)$ and $\mathrm{H}_{\mathrm{Iw}}^{2}\left(U_{K_{\infty}}, \mathcal{O}_{p}(\varrho)\right)$ are finitely generated as $\mathcal{O}_{p}$-modules.

Proof. By Corollary 7.3 we may enlarge $K_{\infty}$ so that $\varrho$ factors through $\mathrm{G}\left(K_{\infty} / \mathbb{Q}\right)$. Further, nothing changes if we then twist by $\varrho^{-1} \varepsilon_{\text {cycl }}$. Hence, we may assume $\varrho=\varepsilon_{\text {cycl }}$.

Set $X=\operatorname{Spec} \mathbb{Z}, S=X-U$. By the theorem of Ferrero-Washington ([Was97, Theorem 7.15]) the module

$$
\lim _{n} \mathcal{O}_{p} \otimes_{\mathbb{Z}} \operatorname{Pic}\left(X_{K_{n}}\right)
$$

is finitely generated over $\mathcal{O}_{p}$. By Proposition 4.2 this is also true for the modules

$$
\lim _{n} \mathrm{H}_{\text {ét }}^{i}\left(S_{K_{n}}, \mathcal{O}_{p}\right) \text {. }
$$

Further, it is an elementary fact of the theory of cyclotomic fields that

$$
\sharp\left(\mathbb{G}_{m}\left(X_{K_{n}}\right) / \mu\left(X_{K_{n}}\right) p_{+} \mathbb{G}_{m}\left(X_{K_{n}}\right)\right) \leq 2,
$$

where $\mu$ denotes the sheaf of unit roots (see [Was97, Theorem 4.12]). In particular, as $p$ was assumed to be an odd prime,

$$
\lim _{n} \mathcal{O}_{p} \otimes_{\mathbb{Z}} p_{-} \mathbb{G}_{m}\left(X_{K_{n}}\right)={\underset{\varliminf}{n}}_{\lim _{n}} \mathcal{O}_{p} \otimes_{\mathbb{Z}} \mu\left(X_{K_{n}}\right) \text {. }
$$

This module is obviously finitely generated over $\mathcal{O}_{p}$ as well. Now use the exact sequence of Proposition 4.1(ii).

After decomposition by characters we may assume that $K_{\infty}$ is a $p$ extension; in particular, totally real. Additionally, we may shrink $U$ by Lemma 7.5 so that $U_{K_{\infty}}$ and $\varrho$ are unramified over $U$. The case that $\varrho$ is 
even has already been settled by the above lemma. The key to the remaining case is the following

Lemma 7.8. Let $K_{\infty}$ be a p-extension, $\varrho$ be odd, and let both be unramified over $U$. Write $\mathfrak{p}$ for the prime ideal of $\Omega$ with $p \in \mathfrak{p}$ and $\operatorname{codim} \mathfrak{p}=1$. Then there exists a non-zero divisor $x$ of $\Omega_{\mathfrak{p}}$ and a quasi-isomorphism

$$
\mathbf{R} \Gamma_{\mathrm{Iw}} / c\left(U_{K_{\infty}}, \mathcal{O}_{p}(\varrho)\right)_{\mathfrak{p}} \cong \Omega_{\mathfrak{p}} / x \Omega_{\mathfrak{p}}[-1] .
$$

Proof. We will first show that $\mathbf{R} \Gamma_{\mathrm{Iw}}\left(U_{K_{\infty}}, \mathcal{O}_{p}(\varrho)\right)$ is quasi-isomorphic to a complex $P^{\bullet}$ of finitely generated projective $\Omega$-modules with $P^{i}=0$ for $i \notin\{1,2\}$. By Proposition 3.4 we can achieve that $P^{i}=0$ for $i>2$.

Recall that in the present situation, $\Omega$ is a local ring. Let $k$ be the residue field of $\Omega$. Proposition 3.5 implies that

$$
k \otimes_{\Omega}^{\mathbb{L}} \mathbf{R} \Gamma_{\mathrm{Iw}}\left(U_{K_{\infty}}, \mathcal{O}_{p}(\varrho)\right)=\mathbf{R} \Gamma_{\text {ét }}\left(U, k \otimes_{\Omega} \operatorname{Ind}_{K_{\infty} / \mathbb{Q}} \mathcal{O}_{p}(\varrho)\right) .
$$

Since $K_{\infty}$ is totally real and $\varrho$ is odd, every lift of the complex conjugation will act by multiplication by -1 on $\operatorname{Ind}_{K_{\infty} / \mathbb{Q}} \mathcal{O}_{p}(\varrho) \otimes_{\Omega} k$; consequently,

$$
\mathrm{H}_{\text {ét }}^{0}\left(U, k \otimes_{\Omega} \operatorname{Ind}_{K_{\infty} / \mathbb{Q}} \mathcal{O}_{p}(\varrho)\right)=0 .
$$

Thus, we can choose $P^{i}=0$ for $i<1$ as well.

Lemma 7.7 then implies that the complex $\mathbf{R} \Gamma_{\mathrm{Iw}}\left(U_{K_{\infty}}, \mathcal{O}_{p}(\varrho)\right)_{\mathfrak{p}}$ is quasiisomorphic to a free $\Omega_{\mathfrak{p}}$-module sitting in degree 1 . The claim follows since $\Omega c\left(U_{K_{\infty}}, \varrho\right)$ is free of rank 1 and $\mathbf{R} \Gamma_{\mathrm{Iw}} / c\left(U_{K_{\infty}}, \mathcal{O}_{p}(\varrho)\right)$ is torsion by Lemma 7.2 .

Putting this and Lemma 7.6 together we see that in the situation of Lemma 7.8, the invertible ideals of the normalisation of $\Omega_{\mathfrak{p}}$ generated by $x^{-1}$, respectively $L\left(U_{K_{\infty}}, \varrho^{-1} \varepsilon_{\text {cycl }}\right)$, agree. But

$$
L\left(U_{K_{\infty}}, \varrho^{-1} \varepsilon_{\text {cycl }}\right)=1
$$

hence, $x$ is unit in the normalisation of $\Omega_{\mathfrak{p}}$ and therefore a unit in $\Omega_{\mathfrak{p}}$ itself. This finishes the proof of Theorem 7.4(i).

Finally, we complete the proof of Theorem 7.4(ii). Let $\left(K_{\infty}, \varrho, U\right)$ be any admissible triple. By Proposition 1.2 it suffices to show that

$$
\operatorname{char} \mathbf{R} \Gamma_{\mathrm{Iw}} / c\left(U_{K_{\infty}}, \mathcal{O}_{p}(\varrho)\right)_{\mathfrak{p}}=L\left(U_{K_{\infty}}, \varrho^{-1} \varepsilon_{\text {cycl }}\right) \Omega_{\mathfrak{p}}
$$

for all prime ideals $\mathfrak{p}$ of codimension 1 . In Lemma 7.6 we have already proved this for those $\mathfrak{p}$ that do not contain $p$. By Theorem 7.4(i) and Proposition 6.7 the equality also holds for the remaining primes.

\section{References}

[Bou89] N. Bourbaki, Commutative Algebra, Elem. Math. (Berlin), Springer, Berlin, 1989. 
[BB05] M. Breuning and D. Burns, Additivity of Euler characteristics in relative algebraic K-groups, Homology Homotopy Appl. 7 (2005), no. 3, 11-36.

[BG03] D. Burns and C. Greither, On the equivariant Tamagawa number conjecture for Tate motives, Invent. Math. 153 (2003), 303-359.

[Eis99] D. Eisenbud, Commutative Algebra. With a View Toward Algebraic Geometry, 3rd ed., Grad. Texts in Math. 150, Springer, New York, NY, 1999.

[Eke90] T. Ekedahl, On the adic formalism, in: The Grothendieck Festschrift, Vol. II, Progr. Math. 87, Birkhäuser, Boston, MA, 1990, 197-218.

[Fla04] M. Flach, The equivariant Tamagawa number conjecture: a survey, in: Stark's Conjectures: Recent Work and New Directions, Contemp. Math. 358, Amer. Math. Soc., Providence, RI, 2004, 79-125.

[HK03] A. Huber and G. Kings, Bloch-Kato conjecture and main conjecture of Iwasawa theory for Dirichlet characters, Duke Math. J. 119 (2003), 393-464.

[Jan88] U. Jannsen, Continuous étale cohomology, Math. Ann. 280 (1988), 207-245.

[KM76] F. Knudsen and D. Mumford, The projectivity of the moduli space of stable curves I, Math. Scand. 39 (1976), 19-55.

[MW84] B. Mazur and A. Wiles, Class fields of abelian extensions of $\mathbb{Q}$, Invent. Math. 76 (1984), 179-330.

[Mil80] J. S. Milne, Étale Cohomology, Princeton Math. Ser. 33, Princeton Univ. Press, Princeton, NJ, 1980.

[Mil86] —, Arithmetic Duality Theorems, Perspect. Math. 1, Academic Press, Boston, MA, 1986.

[Nek03] J. Nekovář, Selmer complexes, preprint, 2nd version, November 2003.

[NSW00] J. Neukirch, A. Schmidt, and K. Wingberg, Cohomology of Number Fields, Grundlehren Math. Wiss. 323, Springer, Berlin, 2000.

[Rub00] K. Rubin, Euler Systems, Ann. Math. Stud. 147, Princeton Univ. Press, Princeton, NJ, 2000.

[Was97] L. C. Washington, Introduction to Cyclotomic Fields, 2nd ed., Grad. Texts in Math. 83, Springer, New York, NY, 1997.

Mathematisches Institut

Universität Leipzig

Postfach 920

D-04109 Leipzig, Germany

E-mail:witte@mathematik.uni-leipzig.de

Received on 10.8.2005

and in revised form on 12.12.2005 